\title{
MULTIPLICITIES OF INDECOMPOSABLE INJECTIVES
}

\author{
AMNON YEKUTIELI AND JAMES J. ZHANG
}

\begin{abstract}
Several results about the multiplicities of indecomposable injectives in the minimal injective resolution of a ring exist in the literature. Mostly these apply to universal enveloping algebras of finite dimensional solvable Lie algebras, and to Gorenstein noetherian PI local rings. We unify these results and extend them to the much wider class of rings with Auslander dualizing complexes.
\end{abstract}

\section{INTRODUCTION}

Dualizing complexes over noncommutative rings were introduced by the first author in 1991 [Ye1]. Since then they have been a very useful tool in studying noncommutative rings. The structure of dualizing complexes is complicated, and is still not well understood, except for some special cases. In this paper we study a numerical invariant of dualizing complexes: the multiplicities of indecomposable injectives in a minimal injective resolution of a dualizing complex.

Assume $A$ is a Gorenstein algebra (i.e. the bimodule $A$ has finite injective dimension on both sides). Then $A$ is a dualizing complex over itself, and we obtain information about the multiplicities of injectives in a minimal injective resolution of $A$. This question has been studied by various authors since the 1980's. Some nice results were proved by Barou-Malliavin [BM], Malliavin [Mal], and BrownLevasseur $[\mathrm{BL}]$ for universal enveloping algebras of finite dimensional solvable Lie algebras; and by Brown [Br], Brown-Hajarnavis [BH] and Stafford-Zhang [SZ] for noetherian PI algebras. The last term of the minimal injective resolution of $A$ was also studied, by the above authors and by Ajitabh-Smith-Zhang [ASZ]. The theme of this paper is to generalize and unify these existing results.

Let $\mathbb{k}$ be a base field. Throughout we shall use the term 'ring' to refer to a unital associative $\mathbb{k}$-algebra. An ' $A$-module' shall mean left module, unless stated otherwise.

Given a left noetherian ring $A$ and a prime ideal $\mathfrak{p} \subset A$ let $E(A / \mathfrak{p})$ denote an injective envelope of the $A$-module $A / \mathfrak{p}$, and let $J(\mathfrak{p})$ denote an indecomposable summand of $E(A / \mathfrak{p})$. So $E(A / \mathfrak{p}) \cong J(\mathfrak{p})^{r}$, where $r:=\operatorname{Grank} A / \mathfrak{p}$, the Goldie rank of $A / \mathfrak{p}$. Recall that $r$ can be calculated as follows: the ring of fractions of $A / \mathfrak{p}$ is isomorphic to a matrix ring $\mathrm{M}_{r}(D)$ over a division ring $D$.

Recall that a minimal injective complex of $A$-modules is a bounded below complex of injectives

$$
I=\left(\cdots \rightarrow 0 \rightarrow I^{i_{0}} \rightarrow \cdots \rightarrow I^{i} \stackrel{\partial^{i}}{\rightarrow} I^{i+1} \rightarrow \cdots\right),
$$

Date: 6 March, 2004.

1991 Mathematics Subject Classification. 16D90, 16E99, 16P40.

Key words and phrases. Dualizing complex, injective module, multiplicity. 
such that for every index $i$ the module of cocycles $\operatorname{Ker}\left(\partial^{i}\right)$ is an essential submodule of $I^{i}$. Given a complex $M \in \mathrm{D}^{+}(\operatorname{Mod} A)$, a minimal injective resolution of $M$ is by definition a quasi-isomorphism $M \rightarrow I$, where $I$ is a minimal injective complex. A minimal injective resolution of $M$ always exists [Ye1, 4.2], and it is unique (up to a non-unique isomorphism of complexes). For a single module $M$ we recover the usual notion of minimal injective resolution.

By 'filtered ring' we mean a ring $A$ equipped with an ascending filtration $\left\{F_{i} A\right\}_{i \in \mathbb{Z}}$ by $\mathbb{k}$-submodules such that $F_{-1} A=0, A=\bigcup F_{i} A$ and $F_{i} A \cdot F_{j} A \subset F_{i+j} A$. The graded ring gr $A$ is called connected if $\operatorname{gr}_{0} A=\mathbb{k}$ and each $\operatorname{gr}_{i} A$ is a finite $\mathbb{k}$ module.

Theorem 0.1. Let $A$ be a filtered ring such that the associated graded ring $\operatorname{gr} A$ is connected, noetherian and has an Auslander balanced dualizing complex. Let $R$ be the rigid dualizing complex of $A$, and let $R \rightarrow I$ be a minimal injective resolution of $R$ as complex of $A$-modules. Then for every prime ideal $\mathfrak{p} \subset A$ the multiplicity $\mu_{i}(\mathfrak{p})$ of $J(\mathfrak{p})$ in $I^{i}$ is

$$
\mu_{i}(\mathfrak{p})= \begin{cases}\operatorname{Grank} A / \mathfrak{p} & \text { if } i=-\operatorname{Cdim}_{R ; A} A / \mathfrak{p} \\ 0 & \text { otherwise. }\end{cases}
$$

The definitions of dualizing complex and related terms (such as the Auslander property) will be reviewed in Section 1. If $R$ is an Auslander dualizing complex over $A$ there is a dimension function $\operatorname{Cdim}_{R ; A}: \operatorname{Mod} A \rightarrow \mathbb{Z}$ called the canonical dimension. For a finite module $M$ the formula is

$$
\operatorname{Cdim}_{R ; A} M:=-\inf \left\{i \mid \operatorname{Ext}_{A}^{i}(M, R) \neq 0\right\} .
$$

Since the hypothesis of the theorem is left-right symmetric (replacing $A$ with its opposite ring $A^{\mathrm{op}}$ ) we also know the multiplicities in the minimal injective resolution of $R$ as complex of $A^{\text {op }}$-modules.

If $A$ is filtered and the graded ring gr $A$ is connected, noetherian and commutative (or somewhat commutative as in Corollary 0.2 below), then gr $A$ has an Auslander balanced dualizing complex, and Cdim $=$ GKdim [YZ1, 6.9]. Therefore we have the following result.

Corollary 0.2. Let $A$ be a filtered ring such that the associated graded ring gr $A$ is connected, noetherian and PI (or FBN, or has enough normal elements in the sense of [YZ1, p. 36]). Let $R$ be a rigid dualizing complex over $A$, and let $R \rightarrow I$ be a minimal injective resolution of $R$ as complex of $A$-modules. Then for every prime ideal $\mathfrak{p} \subset A$ the multiplicity $\mu_{i}(\mathfrak{p})$ of $J(\mathfrak{p})$ in $I^{i}$ is

$$
\mu_{i}(\mathfrak{p})= \begin{cases}\operatorname{Grank} A / \mathfrak{p} & \text { if } i=-\operatorname{GKdim} A / \mathfrak{p}, \\ 0 & \text { otherwise. }\end{cases}
$$

Suppose $A$ is the universal enveloping algebra $U(\mathfrak{g})$ of a finite dimensional Lie algebra $\mathfrak{g}$. Then the associated graded $\operatorname{ring} \operatorname{gr} A$ is commutative, and the rigid dualizing complex of $A$ is $A^{\sigma}[d]$ for some automorphism $\sigma$, where $d:=\operatorname{dim}_{\mathbb{k}} \mathfrak{g}$. Hence:

Corollary 0.3. Let $A:=U(\mathfrak{g})$, the universal enveloping algebra of a Lie algebra $\mathfrak{g}$ with $\operatorname{dim}_{\mathbb{k}} \mathfrak{g}=d<\infty$. Let $A \rightarrow I$ be a minimal injective resolution of $A$ as 
A-module. Then for every prime ideal $\mathfrak{p} \subset A$ the multiplicity $\nu_{i}(\mathfrak{p})$ of $J(\mathfrak{p})$ in $I^{i}$ is

$$
\nu_{i}(\mathfrak{p})= \begin{cases}\operatorname{Grank} A / \mathfrak{p} & \text { if } i=d-\operatorname{GKdim} A / \mathfrak{p}, \\ 0 & \text { otherwise. }\end{cases}
$$

Corollary 0.3 extends a result of Malliavin [Mal, 4.17] and Brown-Levasseur [BL, 6.1], which was proved for enveloping algebras of solvable Lie algebras. Note that our result is valid for any Lie algebra. Corollary 0.2 also applies to factor rings of $U(\mathfrak{g})$ and many quantized algebras listed in [GL].

The universal enveloping algebra of a finite dimensional Lie algebra is Auslander regular of finite global dimension. Most algebras in this paper do not have finite global (or injective) dimension, but often admit Auslander dualizing complexes. Such algebras are generalization of Auslander Gorenstein or Auslander regular algebras. Basic properties of Auslander dualizing complexes and their applications are given in [Ye2], [YZ1], [WZ1].

For FBN rings, every indecomposable injective is of the form $J(\mathfrak{p})$ for some prime ideal $\mathfrak{p}$. In this case we can say more about the structure of dualizing complexes. A version of Theorem 0.4 for non-FBN rings is given in Proposition 3.1.

Theorem 0.4. Let $A$ and $B$ be two FBN rings, and let $R$ be an Auslander, weakly bifinite, Cdim-symmetric dualizing complex over $(A, B)$. Let $R \rightarrow I$ be a minimal injective resolution of $R$ as complex of $A$-modules. Then for every $i$ we have

$$
I^{i} \cong \bigoplus J(\mathfrak{p})^{\mu_{i}(\mathfrak{p})}
$$

where the sum ranges over all prime ideals $\mathfrak{p} \subset A$ with $\operatorname{Cdim}_{R ; A} A / \mathfrak{p}=-i$. The multiplicity $\mu_{i}(\mathfrak{p})$ is a (finite) positive integer.

Theorem 0.4 is a generalization of the results of Brown $[\mathrm{Br}$, Theorem $\mathrm{C}]$, and Stafford-Zhang [SZ, 3.15]. This theorem is part of [YZ2, 4.10]. However, the statement presented here is implicit in [YZ2, 4.10] and the proof we will give is slightly different from the one in [YZ2, 4.10]. The structure of dualizing complexes over FBN rings was studied further in [YZ2] (see Remark 5.4). In some special cases, we are able to compute the multiplicity $\mu_{i}(\mathfrak{p})$ in Theorem 0.4. The following corollary is analogous to [BH, 5.5].

Corollary 0.5. Let $A$ be a noetherian, complete, local, PI $\mathbb{k}$-algebra with injective dimension $d<\infty$ and maximal ideal $\mathfrak{m}$. Suppose that $A / \mathfrak{m}$ is finite over $\mathbb{k}$. Let $A \rightarrow I$ be a minimal injective resolution of $A$ as $A$-module. Then for every $i$ we have an isomorphism

$$
I^{i} \cong \bigoplus J(\mathfrak{p})^{\operatorname{Grank} A / \mathfrak{p}},
$$

where the sum ranges over all prime ideals $\mathfrak{p} \subset A$ with $\operatorname{Kdim} A / \mathfrak{p}=d-i$.

Theorem 0.4 is proved in Section 3, and the other theorems and corollaries are proved in Section 5 .

Acknowledgments. Both authors were supported by the US-Israel Binational Science Foundation. The second author was also supported by the NSF, a Sloan Research Fellowship and the Royalty Research Fund of the University of Washington. Authors also thank the referee for careful reading of the manuscript and valuable comments. 


\section{Review of Definitions}

Let $A$ be a ring (i.e. a $\mathbb{k}$-algebra). As mentioned earlier, we shall usually work with left $A$-modules. We treat right $A$-modules as $A^{\text {op }}$-modules, where $A^{\text {op }}$ is the opposite ring. Bimodules are modules over the enveloping ring $A^{\mathrm{e}}:=A \otimes A^{\mathrm{op}}$, where $\otimes:=\otimes_{\mathbb{k}}$. A finitely generated $A$-module is simply called finite. We refer to [VdB, Ye1, Ye2, YZ1] for basic material on complexes and derived categories, and to $[\mathrm{GW}, \mathrm{MR}$ ] for basic ring theoretic notions such as PI and FBN.

The category of $A$-modules is denoted by $\operatorname{Mod} A$, and its derived category is $\mathrm{D}(\operatorname{Mod} A)$.

We now recall several notions related to dualizing complexes.

Definition 1.1. [Ye1, 3.3], [YZ1, 1.1] Assume $A$ is a left noetherian $\mathbb{k}$-algebra and $B$ is a right noetherian $\mathbb{k}$-algebra. A complex $R \in \mathrm{D}^{\mathrm{b}}\left(\operatorname{Mod} A \otimes B^{\mathrm{op}}\right)$ is called a dualizing complex over $(A, B)$ if it satisfies the following conditions:

(i) $R$ has finite injective dimension over $A$ and $B^{\mathrm{op}}$.

(ii) $R$ has finite cohomology modules over $A$ and $B^{\text {op }}$.

(iii) The canonical morphisms $B \rightarrow \mathrm{RHom}_{A}(R, R)$ in $\mathrm{D}\left(\operatorname{Mod} B^{\mathrm{e}}\right)$, and $A \rightarrow$ $\mathrm{RHom}_{B^{\mathrm{op}}}(R, R)$ in $\mathrm{D}\left(\operatorname{Mod} A^{\mathrm{e}}\right)$, are both isomorphisms.

If moreover $A=B$ we say $R$ is a dualizing complex over $A$.

Whenever we say $R$ is a dualizing complex over $(A, B)$ we tacitly assume that $A$ is left noetherian and $B$ is right noetherian.

Let $R$ be a dualizing complex over $(A, B)$ and let $M$ be a finite $A$-module. The grade of $M$ with respect to $R$ is defined to be

$$
j_{R}(M)=\inf \left\{q \mid \operatorname{Ext}_{A}^{q}(M, R) \neq 0\right\} .
$$

The grade of a $B^{\mathrm{op}}$-module is defined similarly.

Definition 1.2. [Ye2, 1.2], [YZ1, 2.1] A dualizing complex $R$ over $(A, B)$ is called Auslander if

(i) For every finite $A$-module $M$, every integer $q$ and every $B^{\mathrm{op}}$-submodule $N \subset \operatorname{Ext}_{A}^{q}(M, R)$ one has $j_{R}(N) \geq q$.

(ii) The same holds after exchanging $A$ and $B^{\text {op }}$.

The canonical dimension of a finite $A$-module $M$ with respect to the Auslander dualizing complex $R$ is defined to be

$$
\operatorname{Cdim}_{R ; A} M:=-j_{R}(M) \text {. }
$$

By [YZ1, 2.10], $\operatorname{Cdim}_{R ; A}$ is a finitely partitive, exact dimension function (cf. [MR, 6.8.4]). By the left-right symmetry of the situation we obtain a dimension function $\operatorname{Cdim}_{R ; B^{\text {op }}}$ on Mod $B^{\mathrm{op}}$. When there is no danger of confusion we will tend to drop some of the subscripts from the expressions $\operatorname{Cdim}_{R ; A}$ and $\operatorname{Cdim}_{R ; B^{\circ \mathrm{p}}}$.

Definition 1.3. A dualizing complex $R$ over $(A, B)$ is called bifinite (respectively weakly bifinite) if the following conditions hold:

(i) For every $A$-bimodule $M$ which is finite on both sides (respectively, and is a subquotient of $A$ ) the $(A, B)$-bimodule $\operatorname{Ext}_{A}^{q}(M, R)$ is finite on both sides.

(ii) The same holds after exchanging $A$ and $B^{\text {op }}$.

Not every dualizing complex is weakly bifinite (see Example 6.1).

The notion of rigidity is due to Van den Bergh [VdB]. 
Definition 1.4. $[\mathrm{VdB}, 8.1]$ Suppose $R$ is a dualizing complex over $A$. If there is an isomorphism

$$
\rho: R \rightarrow \operatorname{RHom}_{A^{\mathrm{e}}}(A, R \otimes R)
$$

in $\mathrm{D}\left(\operatorname{Mod} A^{\mathrm{e}}\right)$ then we call $(R, \rho)$, or simply $R$, a rigid dualizing complex.

Definition 1.5. [Ye1,4.5] A dualizing complex $R$ over $(A, B)$ is called pre-balanced if

(i) For every simple $A$-module $M$ one has $\operatorname{Ext}_{A}^{i}(M, R)=0$ for all $i \neq 0$, and $\operatorname{Ext}_{A}^{0}(M, R)$ is a simple $B^{\mathrm{op}}$-module.

(ii) The same holds after exchanging $A$ and $B^{\text {op }}$.

Remark 1.6. Pre-balanced dualizing complexes often exist for semilocal or FBN rings. But this is not true in general. For example, the $n$th Weyl algebra, for $n \geq 2$, does not admit any pre-balanced dualizing complexes (see Example 6.5), though it naturally admits an Auslander rigid dualizing complex.

When $A$ is connected graded, there is a notion of balanced dualizing complex introduced in [Ye1]. Let $\mathfrak{m}=A_{>0}$ and let $A^{\prime}$ be the graded vector space dual of $A$. Let $\Gamma_{\mathfrak{m}}$ denote the torsion functor $\lim _{n \rightarrow \infty} \operatorname{Hom}_{A}\left(A / \mathfrak{m}^{n},-\right)$.

Definition 1.7. [Ye1, 4.1] A dualizing complex $R$ over a connected graded ring $A$ is called balanced if there are isomorphisms

$$
\mathrm{R} \Gamma_{\mathfrak{m}}(R) \cong A^{\prime} \cong \mathrm{R} \Gamma_{\mathfrak{m}} \circ(R)
$$

in $\mathrm{D}^{\mathrm{b}}\left(\operatorname{Mod} A^{e}\right)$. A balanced dualizing complex over a local ring is defined similarly [CWZ, Definition 3.7].

Definition 1.8. Suppose we are given a dimension function $\operatorname{dim}_{A}$ on $\operatorname{Mod} A$ and a dimension function $\operatorname{dim}_{B^{\mathrm{op}}}$ on $\operatorname{Mod} B^{\mathrm{op}}$. The pair $\left(\operatorname{dim}_{A}, \operatorname{dim}_{B^{\mathrm{op}}}\right)$ is denoted by dim.

(i) We say that dim is symmetric if for every $(A, B)$-bimodule $M$ finite on both sides there is equality

$$
\operatorname{dim}_{A} M=\operatorname{dim}_{B^{\text {op }}} M .
$$

(ii) In case $A=B$ we say that $\operatorname{dim}$ is weakly symmetric if for every $A$-bimodule $M$ that is a subquotient of $A$ there is equality

$$
\operatorname{dim}_{A} M=\operatorname{dim}_{A^{\text {op }}} M .
$$

(iii) Assume $R$ is an Auslander dualizing complex over $(A, B)$ (respectively over $A$ ). We say $R$ is Cdim-symmetric (respectively Cdim-weakly symmetric) if the pair $\operatorname{Cdim}_{R}:=\left(\operatorname{Cdim}_{R ; A}, \operatorname{Cdim}_{R ; B \text { op }}\right)$ is symmetric (respectively weakly symmetric).

The Gelfand-Kirillov dimension GKdim is always symmetric [MR, 8.3.14(ii)], and Krull dimension Kdim is symmetric for FBN rings [MR, 6.4.13]. It follows from [VdB , 4.8] and [YZ1, 6.22] that rigid Auslander dualizing complexes over filtered algebras are Cdim-weakly symmetric. A similar statement holds in the semilocal case [WZ1, 0.1]. But Example 6.2 shows that not every Auslander bifinite dualizing complex is Cdim-weakly symmetric. 


\section{Preliminary Results}

In this section we collect some preliminary results about the pre-balanced condition, bifiniteness and so on.

Lemma 2.1. The following dualizing complexes are bifinite or weakly bifinite.

(1) If $A$ and $B$ are noetherian PI algebras, then every dualizing complex $R$ over $(A, B)$ is bifinite.

(2) If $A$ is filtered and gr $A$ is noetherian with a balanced dualizing complex, then the rigid dualizing complex over $A$ is weakly bifinite.

(3) Let $(A, \mathfrak{m})$ and $(B, \mathfrak{n})$ be noetherian complete semilocal algebras such that there is a Morita duality between them. Suppose that

(a) $\Gamma_{\mathfrak{m}}$ and $\Gamma_{\mathfrak{n}} \circ$ have finite cohomogical dimension,

(b) $A$ and $B$ satisfy the $\chi$-condition, and

(c) $A / \mathfrak{m}$ is $P I$.

Then every pre-balanced dualizing complex over $(A, B)$ is bifinite.

Proof. (1) Let $M$ be an $A$-bimodule finite on both sides. It follows from the properties of dualizing complexes that $\operatorname{Ext}_{A}^{i}(M, R)$ is a finite $B^{\text {op }}$-module. It remains to show that it is finite over $A$. So we may forget the $B^{\mathrm{op}}$-module structure of $R$ and by the long exact sequence we may replace $R$ by a finite $A$-module. Then the result follows from $[\mathrm{SZ}, 3.5]$.

(2) This follows from [YZ1, 6.21]. (However we don't know if $R$ is bifinite in this case.)

(3) This follows from [CWZ, 0.1(4)]. For the definitions and some details of the conditions listed, see [CWZ, WZ1].

The pre-balanced condition is a natural one according to [CWZ]. From the next lemma we see that it is automatic for local rings.

Lemma 2.2. Let $R$ be a weakly bifinite dualizing complex over $(A, B)$. Suppose that for every simple $B^{\mathrm{op}}$-module $S$ the ring $B / \operatorname{Ann}_{B^{\mathrm{op}}}(S)$ is simple artinian.

(1) Condition 1.5(i) implies 1.5(ii). Consequently, $R$ is pre-balanced if and only 1.5(i) holds.

(2) If $A$ is local then $B$ is local too, and $R[d]$ is pre-balanced for some integer $d$.

Proof. (1) Suppose that $1.5(\mathrm{i})$ holds. Let $S$ be a simple $B^{\text {op }}$-module and $\mathfrak{n}:=$ $\operatorname{Ann}_{B^{\text {op }}}(S)$. By hypothesis, $B / \mathfrak{n}$ is simple artinian, and hence $B / \mathfrak{n} \cong S^{r}$ as $B^{\text {op }}$ modules. Since $R$ is weakly bifinite, $M:=\operatorname{Ext}_{B_{\text {op }}}^{j}(B / \mathfrak{n}, R)$ is noetherian on both sides. It is a right $(B / \mathfrak{n})$-module, so it's right artinian. By Lenagan's lemma [GW, 7.10], $M$ is also left artinian. It follows that $A / \operatorname{Ann}_{A}(M)$ is an $A$-submodule of a finite direct sum of copies of $M$, which is left artinian. By 1.5(i),

$$
\operatorname{Ext}_{A}^{i}\left(\operatorname{Ext}_{B^{\circ \mathrm{op}}}^{j}(B / \mathfrak{n}, R), R\right)=\operatorname{Ext}_{A}^{i}(M, R)=0
$$

for all $i \neq 0$. Then the double-Ext spectral sequence [YZ1, 1.7] shows that $\operatorname{Ext}_{B^{\text {op }}}^{j}(B / \mathfrak{n}, R)=0$ for all $j \neq 0$ and that

$$
B / \mathfrak{n} \cong \operatorname{Ext}_{A}^{0}\left(\operatorname{Ext}_{B^{\text {op }}}^{0}(B / \mathfrak{n}, R), R\right) .
$$

Hence $\operatorname{Ext}_{B^{\text {op }}}^{j}(S, R)=0$ for all $j \neq 0$ and

$$
S \cong \operatorname{Ext}_{A}^{0}\left(\operatorname{Ext}_{B^{\mathrm{op}}}^{0}(S, R), R\right) .
$$


It follows from 1.5(i) that the length of the $A$-module $\operatorname{Ext}_{B^{\text {op }}}^{0}(S, R)$ is equal to the length of the $B^{\text {op }}$-module $\operatorname{Ext}_{A}^{0}\left(\operatorname{Ext}_{B \text { op }}^{0}(S, R), R\right)$, which is one. Therefore $\operatorname{Ext}_{B^{\circ \mathrm{p}}}^{0}(S, R)$ is simple.

(2) Let $\mathfrak{m}$ be the maximal ideal of $A$. Shift $R$ so that $\operatorname{Ext}_{A}^{0}(A / \mathfrak{m}, R) \neq 0$. By weak finiteness and Lenagan's lemma as in the proof of $(1), \operatorname{Ext}_{A}^{i}(A / \mathfrak{m}, R)$ is artinian on both sides for all $i$. Let

$$
i_{0}:=\min \left\{i \mid \operatorname{Ext}_{A}^{i}(A / \mathfrak{m}, R) \neq 0\right\}, \quad i_{1}:=\max \left\{i \mid \operatorname{Ext}_{A}^{i}(A / \mathfrak{m}, R) \neq 0\right\} .
$$

By induction on length one sees that for all finite length $A$-modules $M$

$$
i_{0}=\min \left\{i \mid \operatorname{Ext}_{A}^{i}(M, R) \neq 0\right\}, \quad i_{1}=\max \left\{i \mid \operatorname{Ext}_{A}^{i}(M, R) \neq 0\right\} .
$$

Let $S$ be a simple $B^{\text {op }}$-module. By our hypothesis $\mathfrak{n}:=\operatorname{Ann}_{B^{\text {op }}}(S)$ is an ideal such that $B / \mathfrak{n}$ is simple artinian. For each $j$ let $M_{j}:=\operatorname{Ext}_{B^{\text {op }}}^{j}(B / \mathfrak{n}, R)$. Then by weak finiteness and Lenagan's lemma $M_{j}$ is artinian on both sides. Let

$$
j_{0}:=\min \left\{j \mid M_{j} \neq 0\right\}, \quad j_{1}:=\max \left\{j \mid M_{j} \neq 0\right\} .
$$

Then $\operatorname{Ext}_{A}^{i}\left(M_{j}, R\right) \neq 0$ for $i=i_{0}, i_{1}$ and $j=j_{0}, j_{1}$. Note that these are four corner terms in the $E_{2}$-page of the double-Ext spectral sequence [YZ1, 1.7]

$$
E_{2}^{i j}:=\operatorname{Ext}_{A}^{i}\left(\operatorname{Ext}_{B^{\text {op }}}^{j}(B / \mathfrak{n}, R), R\right) \Rightarrow B / \mathfrak{n} .
$$

Since the terms $E_{2}^{i_{0} j_{1}}$ and $E_{2}^{i_{1} j_{0}}$ will survive in the $E_{\infty}$-page, these two terms must be on the diagonal, namely $i_{0}=j_{1}$ and $i_{1}=j_{0}$. This shows that $i_{0}=i_{1}=j_{0}=j_{1}$, and all are equal to 0 because $i_{0} \leq 0 \leq i_{1}$. As a consequence $\operatorname{Ext}_{B^{\circ}}^{j}(S, R)=0$ for all $j \neq 0$, and $\operatorname{Ext}_{B^{\text {op }}}^{0}(S, R)$ is a nonzero artinian $A$-module. Therefore $\operatorname{Ext}_{A}^{0}(-, R)$ and $\operatorname{Ext}_{B^{\text {op }}}^{0}(-, R)$ are a duality between the category of finite length $A$-modules and that of finite length $B^{\text {op }}$-modules. Therefore $B$ is local and $R$ is pre-balanced.

Recall from [WZ2] that $A$ satisfies the Nakayama condition if for every nonzero finite right $A$-module $N$ there is a (simple) left $A$-module $S$ such that $N \otimes_{A} S \neq 0$. It is easily seen that noetherian semilocal rings and FBN rings satisfy the Nakayama condition. One can also verify that the first Weyl algebra, which is neither FBN nor semilocal, satisfies the Nakayama condition.

The following Proposition is an ungraded version of [Ye1, 3.9].

Proposition 2.3. Let $R_{1}$ and $R_{2}$ be pre-balanced dualizing complexes over $(A, B)$. If $A$ satisfies the Nakayama condition, then there is an invertible A-bimodule $L$ such that $R_{1} \cong L \otimes_{A} R_{2}$.

To prove this we need to use two-sided tilting complexes (see [Ri1, Ri2, Ye3]). A two-sided tilting complex $T \in \mathrm{D}^{\mathrm{b}}\left(\operatorname{Mod} B \otimes A^{\mathrm{op}}\right)$ is called pre-balanced if for every simple $A$-module $S$ and every $i \neq 0$ one has $\mathrm{H}^{i}\left(T \otimes_{A}^{\mathrm{L}} S\right)=0$.

Lemma 2.4. If A satisfies the Nakayama condition, then a two-sided tilting complex $T \in \mathrm{D}^{\mathrm{b}}\left(\operatorname{Mod} B \otimes A^{\mathrm{op}}\right)$ is pre-balanced if and only if it is isomorphic to an invertible bimodule.

Proof. If $T$ is isomorphic to an invertible bimodule, then it is clearly pre-balanced. So we only need to prove the other implication. 
Let $i_{1}:=\max \left\{i \mid \mathrm{H}^{i} T \neq 0\right\}$. Since $\mathrm{H}^{i_{1}} T$ is a finite $A^{\mathrm{op}}$-module, by the Nakayama condition there is a simple $A$-module $S$ such that $\left(\mathrm{H}^{i_{1}} T\right) \otimes_{A} S \neq 0$. By the Künneth spectral sequence (cf. [Ye3, 2.1]) there is an isomorphism

$$
\left(\mathrm{H}^{i_{1}} T\right) \otimes_{A} S \cong \mathrm{H}^{i_{1}}\left(T \otimes_{A}^{\mathrm{L}} S\right) .
$$

Since $T$ is pre-balanced we see that $i_{1}=0$.

We conclude that $\left(\mathrm{H}^{0} T\right) \otimes_{A} S \neq 0$ for all simple $A$-modules $S$. Therefore also $\left(\mathrm{H}^{0} T\right) \otimes_{A} M \neq 0$ for every nonzero finite $A$-module $M$.

Proceeding exactly as in $[\mathrm{Ye} 3,2.3]$ we conclude that $T \cong \mathrm{H}^{0} T$ is an invertible bimodule.

Proof of Proposition 2.3. First we note that since for $i=1,2$ the complex $R_{i}$ is pre-balanced, the functor $M \mapsto \operatorname{Ext}_{A}^{0}\left(M, R_{i}\right)$ is a duality between the category of finite length $A$-modules and that of finite length $B^{\mathrm{op}}$-modules. Furthermore, for every finite length $A$-module $M, \operatorname{RHom}_{A}\left(M, R_{i}\right) \cong \operatorname{Ext}_{A}^{0}\left(M, R_{i}\right)$; and likewise for $B^{\mathrm{op}}$-modules.

By [Ye1, 3.9 and 3.10] there is a two-sided tilting complex $T$ over $A$ such that $R_{2} \cong T \otimes_{A}^{\mathrm{L}} R_{1}$, and such that

$$
\left.\operatorname{RHom}_{B^{\circ \mathrm{p}}}\left(\operatorname{RHom}_{A}\left(M, R_{1}\right), R_{2}\right)\right) \cong T \otimes_{A}^{\mathrm{L}} M
$$

for all $M \in \mathrm{D}_{\mathrm{f}}^{\mathrm{b}}(\operatorname{Mod} A)$. Taking $M$ to be a simple $A$-module we get $\mathrm{H}^{i}\left(T \otimes_{A}^{\mathrm{L}} M\right)=0$ if $i \neq 0$. Thus $T$ is pre-balanced, and by Lemma 2.4 it is isomorphic to an invertible bimodule.

Let $\mathfrak{p}$ be a prime ideal of $A$. We denote by $J(\mathfrak{p})$ the indecomposable injective module associated to $\mathfrak{p}$, i.e. the $A$-injective envelope of a uniform left ideal of $A / \mathfrak{p}$.

Given an $(A / \mathfrak{p})$-module $M$ its reduced rank (at the prime $\mathfrak{p}$ ) is

$$
\text { red.rank } M:=\operatorname{Grank}(M / \tau(M)),
$$

where $\tau(M)$ denotes the torsion submodule of $M$ and Grank denotes Goldie rank. If $Q$ is the ring of fractions of $A / \mathfrak{p}$ then red.rank $M$ equals the length of $Q \otimes_{A} M$ as $Q$-module.

A complex $I$ of $A$-modules is called a minimal injective complex if (a) each term $I^{i}$ is $A$-injective and (b) each ker $\partial^{i}$ is an essential submodule of $I^{i}$ where $\partial^{i}: I^{i} \rightarrow I^{i+1}$ is the $i$ th coboundary map. The next lemma is [YZ2, 4.12], which is a generalization of $[\mathrm{Br}, 2.3]$.

Lemma 2.5. [YZ2, 4.12] Suppose $A$ is a left noetherian ring. Let I be a minimal injective complex of $A$-modules. Let $\mathfrak{p}$ be a prime ideal of $A$, and let $\mu_{i}(\mathfrak{p})$ be the multiplicity of $J(\mathfrak{p})$ in $I^{i}$. Then

(1) The image of the map

$$
\operatorname{Hom}_{A}\left(A / \mathfrak{p}, \partial^{i-1}\right): \operatorname{Hom}_{A}\left(A / \mathfrak{p}, I^{i-1}\right) \rightarrow \operatorname{Hom}_{A}\left(A / \mathfrak{p}, I^{i}\right)
$$

is a torsion $(A / \mathfrak{p})$-module.

(2) There is equality

$$
\mu_{i}(\mathfrak{p})=\text { red.rank } \operatorname{Hom}_{A}\left(A / \mathfrak{p}, I^{i}\right)=\operatorname{red} . \operatorname{rank} \operatorname{Ext}_{A}^{i}(A / \mathfrak{p}, I) .
$$

(3) Let $\mathfrak{p}$ and $\mathfrak{q}$ be two primes of $A$ and $M$ an $(A / \mathfrak{p})-(A / \mathfrak{q})$-bimodule. Assume $M$ is nonzero, torsion-free as $(A / \mathfrak{q})^{\mathrm{op}}$-module and finite non-torsion as $(A / \mathfrak{p})$-module. If $\mu_{i}(\mathfrak{p}) \geq 1$ then $\operatorname{Ext}_{A}^{i}(M, I)$ is a non-torsion $(A / \mathfrak{q})$ module. 


\section{Multiplicity, Part I}

In this section $R$ is a dualizing complex over a pair of rings $(A, B)$, and $R \rightarrow I$ is a minimal injective resolution of $R$ as complex of $A$-modules. For a prime ideal $\mathfrak{p} \subset A$ and an integer $i$ let $\mu_{i}(\mathfrak{p})$ be the multiplicity of $J(\mathfrak{p})$ in $I^{i}$. Using Lemma 2.5 we are able to compute the multiplicities $\mu_{i}(\mathfrak{p})$ in some cases.

Proposition 3.1. Suppose $R$ is a weakly bifinite dualizing complex over $(A, B)$. Then $\mu_{i}(\mathfrak{p})$ is finite for all $i$ and all primes $\mathfrak{p} \subset A$. If moreover $R$ is Auslander and Cdim-symmetric, then $\mu_{i}(\mathfrak{p})$ is nonzero if and only if $i=-\operatorname{Cdim}_{R ; A} A / \mathfrak{p}$.

Proof. Because $R$ is weakly bifinite, $\operatorname{Ext}_{A}^{i}(A / \mathfrak{p}, R)$ is a finite $A / \mathfrak{p}$-module. Therefore red.rank ${ }_{A / \mathfrak{p}} \operatorname{Ext}_{A}^{i}(A / \mathfrak{p}, R)$ is finite. The first assertion follows from Lemma $2.5(2)$.

To prove the second assertion let $j:=j_{R}(A / \mathfrak{p})=-\operatorname{Cdim}_{R ; A} A / \mathfrak{p}$. By [YZ1, 1.11] we know that $\mu_{i}(\mathfrak{p}) \neq 0$ for some $i$. So it suffices to show that $\mu_{i}(\mathfrak{p})=0$ for all $i \neq j$. But when $i \neq j$ we have

$$
\operatorname{Cdim}_{R ; B^{\text {op }}} \operatorname{Ext}_{A}^{i}(A / \mathfrak{p}, R)<\operatorname{Cdim}_{R ; B^{\text {op }}} \operatorname{Ext}_{A}^{j}(A / \mathfrak{p}, R)=-j .
$$

Hence $\operatorname{Ext}_{A}^{i}(A / \mathfrak{p}, R)$ is a torsion $(A / \mathfrak{p})$-module. The assertion now follows from Lemma 2.5(2).

Proof of Theorem 0.4. First of all since $A$ is noetherian, every injective module is a direct sum of indecomposable injectives. Since $A$ is FBN, every indecomposable injective is of form $J(\mathfrak{p})$ for some prime ideal $\mathfrak{p} \subset A$. By Proposition 3.1, $\mu_{i}(\mathfrak{p})$ is finite, and it is nonzero if and only if $i=-\operatorname{Cdim}_{R ; A} A / \mathfrak{p}$.

[SZ, 3.15] is an almost immediate consequence:

Corollary 3.2. [SZ, 3.15] Suppose A is a noetherian, AS-Gorenstein, PI algebra. Then the ith term of the minimal injective resolution of $A$ as complex of $A$-modules is isomorphic to

$$
\bigoplus J(\mathfrak{p})^{\nu_{i}(\mathfrak{p})}
$$

where the sum ranges over all prime ideals $\mathfrak{p}$ such that $\operatorname{Kdim} A / \mathfrak{p}=\operatorname{Kdim} A-i$. The multiplicity $\nu_{i}(\mathfrak{p})$ is a (finite) positive integer.

Proof. Since $A$ is AS-Gorenstein and PI it follows from [SZ, 3.10] that $A$ is Auslander Gorenstein and Kdim-Macaulay. Let $d$ be the injective dimension of $A$, which equals its Krull dimension. Then $R:=A[d]$ is an Auslander, bifinite, Cdim-symmetric dualizing complex over $A$. Now use Proposition 3.1, noting that $\nu_{i}(\mathfrak{p})=\mu_{i-d}(\mathfrak{p})$.

If $A$ is prime the multiplicities $\mu_{i}(\mathfrak{o})$ for the zero ideal $\mathfrak{o}$ can be computed, as we now show. But first a bit of notation. Say $M$ is an $A$-B-bimodule. We write $\operatorname{Grank}_{A} M$ for the Goldie rank of $M$ considered as $A$-module, and likewise Grank $_{B^{\circ \mathrm{p}}} M$.

Proposition 3.3. Suppose $A$ and $B$ are prime rings and $R$ is an Auslander dualizing complex over $(A, B)$. Let $H^{-d} R$ be the lowest nonzero cohomology of $R$.

(1) Let $Q(A)$ be the ring of fractions of $A$, and likewise $Q(B)$. Then

$$
Q(A) \otimes_{A} H^{i} R \cong H^{i} R \otimes_{B} Q(B)
$$

as $A$-B-bimodules for all $i$, and they are 0 for $i \neq-d$. 
(2) The bimodule $H^{-d}(R)$ is torsion-free on both sides,

$$
\operatorname{Grank}_{A} H^{-d} R=\operatorname{Grank}_{B^{\text {op }}} B
$$

and

$$
\operatorname{Grank}_{B^{\text {op }}} H^{-d} R=\operatorname{Grank}_{A} A .
$$

(3) Let $\mathfrak{o}$ be the zero ideal of $A$. Then

$$
\begin{aligned}
& \qquad \mu_{-d}(\mathfrak{o})=\operatorname{Grank}_{B^{\mathrm{op}}} B \\
& \text { and } \mu_{i}(\mathfrak{o})=0 \text { for all } i \neq-d .
\end{aligned}
$$

Proof. (1) We may assume that $R^{-d-1}=0$. Hence $\operatorname{Ext}_{A}^{-d}(-, R)=\operatorname{Hom}_{A}(-, H)$ where $H:=\mathrm{H}^{-d}(R)$. Since $A$ and $B$ are prime of Cdim $=d$, every torsion module $M$ has $\operatorname{Cdim} M<d$. Thus, if $M$ is a torsion finite $A$-module, then $\operatorname{Hom}_{A}(M, H)=$ 0 . Hence $H$ is torsion-free on the left; and by symmetry also on the right. Let $b \in B$ be a regular element. Then right multiplication by $b$ on $Q(A) \otimes_{A} H$ is injective. Since this is a finite length $Q(A)$-module, $b$ has to act invertibly. Hence

$$
Q(A) \otimes_{A} H \cong Q(A) \otimes_{A} H \otimes_{B} Q(B) .
$$

By symmetry we also get

$$
H \otimes_{B} Q(B) \cong Q(A) \otimes_{A} H \otimes_{B} Q(B) .
$$

By the Auslander property, for every $i>-d$ we have

$$
\operatorname{Cdim}_{R ; A} \mathrm{H}^{i} R=\operatorname{Cdim}_{R ; A} \operatorname{Ext}_{A}^{i}(A, R) \leq-i<d=\operatorname{Cdim}_{R ; A} A .
$$

If $i<-d$, then the Auslander property shows that $\mathrm{H}^{i} R=0$. This implies that

$$
Q(A) \otimes H^{i} R=H^{i} R \otimes Q(B)=0
$$

for all $i \neq-d$.

(2) By the proof of part 1, H= $\mathrm{H}^{-d} R$ is torsion-free on both sides and

$$
Q(A) \otimes_{A} H \cong Q(A) \otimes_{A} H \otimes_{B} Q(B) \cong H \otimes_{B} Q(B) .
$$

By $[\mathrm{YZ1}, 2.15]$ the functor $\operatorname{Ext}_{A}^{-d}(-, R)$ is a duality between the subquotient category $\mathrm{M}_{d, \mathrm{f}} / \mathrm{M}_{d-1, \mathrm{f}}$ of $\operatorname{Mod} A$ and the subquotient category $\mathrm{M}_{d, \mathrm{f}}^{\mathrm{op}} / \mathrm{M}_{d-1, \mathrm{f}}^{\mathrm{op}}$ of $\operatorname{Mod} B^{\mathrm{op}}$. (For the definition of the category $\mathrm{M}_{d, \mathrm{f}}$ see $\left[\mathrm{YZ1}\right.$, Section 2].) Now $\mathrm{M}_{d, \mathrm{f}} / \mathrm{M}_{d-1, \mathrm{f}} \approx$ $\operatorname{Mod}_{\mathrm{f}} Q(A)$, and similarly for $B^{\mathrm{op}}$. We conclude that

$$
\operatorname{Hom}_{Q(A)}\left(-, Q(A) \otimes_{A} H \otimes_{B} Q(B)\right): \operatorname{Mod}_{\mathrm{f}} Q(A) \rightarrow \operatorname{Mod}_{\mathrm{f}} Q(B)^{\mathrm{op}}
$$

is a duality. Since for any torsion-free $A$-module $M$ the Goldie $\operatorname{rank} \operatorname{Grank}_{A} M$ coincides with the length of $Q(A) \otimes_{A} M$ in $\operatorname{Mod} Q(A)$, and likewise for $B^{\text {op }}$, we are finished.

(3) The assertion follows from Lemma 2.5(2) and part (2). 


\section{The Trace Property}

To prove the results stated in the introduction we need to extend the formula in Proposition 3.3(3) to all prime ideals. First we need to construct a correspondence between the set of the prime ideals of $A$ and that of $B$. With this correspondence we show that the multiplicity $\mu(\mathfrak{p})$ for $\mathfrak{p} \subset A$ can be computed by the Goldie rank of $B / \mathfrak{q}$ for the corresponding prime $\mathfrak{q} \subset B$.

Definition 4.1. Suppose that $A \rightarrow \bar{A}$ and $B \rightarrow \bar{B}$ are algebra homomorphisms such that $\bar{A}$ is a finite left $A$-module and $\bar{B}$ is a finite right $B$-module. Also suppose $R$ and $\bar{R}$ are dualizing complexes over $(A, B)$ and $(\bar{A}, \bar{B})$ respectively. We say $R$ induces $\bar{R}$ if there exists a pair of morphisms

$$
\operatorname{Tr}, \operatorname{Tr}^{\text {op }}: \bar{R} \rightarrow R
$$

in $\mathrm{D}\left(\operatorname{Mod} A \otimes B^{\mathrm{op}}\right)$, such that the induced morphisms $\bar{R} \rightarrow \operatorname{RHom}_{A}(\bar{A}, R)$ in $\mathrm{D}\left(\operatorname{Mod} \bar{A} \otimes B^{\mathrm{op}}\right)$ and $\bar{R} \rightarrow \mathrm{RHom}_{B^{\mathrm{op}}}(\bar{B}, R)$ in $\mathrm{D}\left(\operatorname{Mod} A \otimes \bar{B}^{\text {op }}\right)$ are both isomorphisms. The morphism $\operatorname{Tr}$ is called a left trace, and $\operatorname{Tr}^{\mathrm{op}}$ is called a right trace.

It might happen that $\operatorname{Tr}=\operatorname{Tr}^{\text {op }}$ (cf. the rigid trace in [YZ1]), but we do not require this.

The following two lemmas are proved in [YZ1, 3.9]. (The rigidity mentioned in [YZ1, 3.9] is not used in the proof.)

Lemma 4.2. Suppose $R$ and $\bar{R}$ are dualizing complexes over $(A, B)$ and $(\bar{A}, \bar{B})$ respectively, and $\bar{R}$ is induced by $R$. Then for every $\bar{A}$-module $M$ one has

$$
\operatorname{RHom}_{A}(M, R) \cong \operatorname{RHom}_{\bar{A}}(M, \bar{R})
$$

in $\mathrm{D}\left(\operatorname{Mod} B^{\mathrm{op}}\right)$. The same holds after exchanging $A$ and $B^{\mathrm{op}}$.

Lemma 4.3. Let $R$ be an Auslander dualizing complex over $(A, B)$. Suppose $\bar{R}$ is a dualizing complex over $(\bar{A}, \bar{B})$ induced by $R$. Then:

(1) $\bar{R}$ is Auslander.

(2) For every $\bar{A}$-module $M$ one has $\operatorname{Cdim}_{R ; A} M=\operatorname{Cdim}_{\bar{R} ; \bar{A}} M$; and likewise after replacing $A$ with $B^{\mathrm{op}}$.

Definition 4.4. A dualizing complex $R$ over $(A, B)$ is said to have the trace property for ideals if the following conditions hold:

(i) There is an inclusion-preserving bijection $\phi$ between the lattice of two-sided ideals of $A$ and the lattice of two-sided ideals of $B$.

(ii) Given an ideal $\mathfrak{a} \subset A$ let $\mathfrak{b}:=\phi(\mathfrak{a}), \bar{A}:=A / \mathfrak{a}$ and $\bar{B}:=B / \mathfrak{b}$. Then there is a dualizing complex $\bar{R}$ over $(\bar{A}, \bar{B})$ that is induced by $R$.

If these conditions hold for the set of prime ideals (instead of the set of all ideals) then $R$ is said to have the trace property for prime ideals.

The trace property for ideals is not automatic (see Examples 6.1 and 6.2). But this property can be proved for several classes of dualizing complexes.

Proposition 4.5. The following dualizing complexes have the trace properties for ideals.

(1) Suppose $A$ is a filtered algebra and gr $A$ is noetherian connected with balanced dualizing complex. Then the rigid dualizing complex over $A$ has the trace property for ideals. The lattice isomorphism $\phi$ is the identity on the set of ideals of $A$. 
(2) Let $A$ and $B$ be algebras satisfying the hypotheses in Lemma 2.1(3). Then every pre-balanced dualizing complex over $(A, B)$ has the trace property for ideals, and the lattice isomorphism $\phi$ is the one given in [AF, 24.6] (and in [CWZ, 5.7]).

(3) Let $A$ be a complete semilocal algebra with $A / \mathfrak{m}$ finite over $\mathbb{k}$ for every maximal ideal $\mathfrak{m}$. Let $R$ be the balanced dualizing complex over $A$ (see $[\mathrm{CWZ}$, Definition 3.7]). Then $R$ has the trace property for ideals. The lattice isomorphism $\phi$ is the identity on the set of ideals of $A$.

Proof. (1) This follows from [YZ1, 6.17].

(2), (3) These assertions follow from [CWZ, 5.6, 5.7 and 5.9].

To compute multiplicities of injectives we only need the trace property for prime ideals.

Lemma 4.6. Let $R$ be an Auslander dualizing complex over $(A, B)$. If $R$ has the trace property for ideals then it has the trace property for prime ideals.

Proof. Suppose $\phi$ is a lattice isomorphism between the set of all ideals of $A$ and the set of all ideals of $B$ as in Definition 4.4(1). We only need to show that the restriction of $\phi$ is a bijection between the set of prime ideals of $A$ and the set of prime ideals of $B$. By symmetry, it suffices to show that if $\mathfrak{a} \subset A$ is prime then $\mathfrak{b}:=\phi(\mathfrak{a}) \subset B$ is prime.

Suppose $\mathfrak{b}$ is not prime. Let $\bar{A}:=A / \mathfrak{a}$ and $\bar{B}:=B / \mathfrak{b}$ be the quotient rings, and let $\bar{R}$ be the induced dualizing complex. By Lemma $4.3 \bar{R}$ is also Auslander. Suppose $\operatorname{Cdim}_{\bar{R} ; \bar{A}} \bar{A}=q$. Then of course $\operatorname{Cdim}_{\bar{R} ; \bar{B}^{\text {op }}} \bar{B}=q$. Since $\mathfrak{b}$ is not prime, there is a prime ideal $\mathfrak{q} \supsetneqq \mathfrak{b}$ such that $\operatorname{Cdim}_{\bar{R} ; \bar{B}^{\text {op }}} \bar{B} / \mathfrak{q}=q$. Suppose $\mathfrak{p} \subset A$ is the ideal corresponding to $\mathfrak{q}$. Then $\mathfrak{p} \supsetneqq \mathfrak{a}$ and $\operatorname{Cdim}_{\bar{R} ; \bar{A}} \bar{A} / \mathfrak{p}=q$. This is impossible because $A / \mathfrak{a}$ is prime of $\operatorname{Cdim}=q$.

\section{Multiplicity, Part II}

In this section we prove Theorems 0.1 and 0.2 and Corollaries 0.3 and 0.5. Recall that Grank $M$ denotes the Goldie rank of a module $M$.

Theorem 5.1. Let $R$ be an Auslander dualizing complex over a pair of rings $(A, B)$. Assume $R$ has the trace property for prime ideals. Let $R \rightarrow I$ be a minimal injective resolution of $R$ as a complex of $A$-modules. Let $\mathfrak{p}$ be a prime ideal of $A$, and let $\mathfrak{q}$ be the corresponding prime ideal of $B$. Denote by $\mu_{i}(\mathfrak{p})$ be the multiplicity of the indecomposable injective module $J(\mathfrak{p})$ in $I^{i}$. Then

$$
\mu_{i}(\mathfrak{p})= \begin{cases}\operatorname{Grank} B / \mathfrak{q} & \text { if } i=-\operatorname{Cdim}_{R} A / \mathfrak{p} \\ 0 & \text { otherwise. }\end{cases}
$$

Proof. By the trace property for prime ideals and Lemma 4.3 we reduce to the case when $\mathfrak{p}=0$. Then the assertion follows from Proposition 3.3(3).

Recall that the trace property holds for several classes of algebras (see Proposition 4.5). Also note that the Auslander condition is quite natural; cf. [YZ1].

Proof of Theorem 0.1. This follows from Theorem 5.1 and Proposition 4.5(1). 
Proof of Corollary 0.2. If gr $A$ is noetherian, and if it is FBN (or PI) or has enough normal elements, then $A$ has a rigid Auslander GKdim-Macaulay dualizing complex $R$ [YZ1, 6.9]. By definition, GKdim-Macaulay means that $\operatorname{Cdim}_{R}=$ GKdim. Thus the assertion follows from Theorem 0.1.

Corollary 5.2. Let $A$ be a filtered ring such that the associated graded ring gr $A$ is connected graded, noetherian and Auslander Gorenstein. Let $\nu_{i}(\mathfrak{p})$ denote the multiplicity of $J(\mathfrak{p})$ in the ith term of a minimal injective resolution of $A$ as left A-module. Then

$$
\nu_{i}(\mathfrak{p})= \begin{cases}\operatorname{Grank} A / \mathfrak{p} & \text { if } i=\operatorname{Cdim} A-\operatorname{Cdim} A / \mathfrak{p}, \\ 0 & \text { otherwise. }\end{cases}
$$

Here $\operatorname{Cdim}:=\operatorname{Cdim}_{R}$, where $R$ is the rigid dualizing complex over $A$, which is Auslander.

Proof. By [YZ1, 6.18] we know that $R \cong A^{\sigma}[n]$, where $\sigma$ is a filtered algebra automorphism of $A$. Then $n=\operatorname{Cdim} A$, and $R \cong A[n]$ in $\mathrm{D}(\operatorname{Mod} A)$. Thus the assertion follows from Theorem 0.1.

Proof of Corollary 0.3. The graded ring gr $U(\mathfrak{g})$ is commutative. By [YZ1, 6.9] one has $\operatorname{Cdim}=$ GKdim. It is also clear that $\operatorname{GKdim} U(\mathfrak{g})=\operatorname{dim}_{\mathbb{k}} \mathfrak{g}$. Therefore the assertion follows from Corollary 5.2.

Applying Theorem 5.1 to the FBN rings we obtain the following result. Note that there might not exist a dualizing complex over $\mathrm{FBN}$ rings $(A, B)$ (even $A=B$ ). If it exists, the dualizing complex may not have the Auslander property and the trace property [Example 6.1].

Corollary 5.3. Let $A$ and $B$ be FBN rings, and let $R$ be an Auslander dualizing complex over $(A, B)$ with the trace property for prime ideals. Let $R \rightarrow I$ be a minimal injective resolution of $R$ as complex of $A$-modules. Then for every integer $i$ there is a decomposition

$$
I^{i} \cong \bigoplus J(\mathfrak{p})^{\mu_{i}(\mathfrak{p})},
$$

where the sum ranges over all prime ideals $\mathfrak{p} \subset A$ with $\operatorname{Cdim}_{R} A / \mathfrak{p}=-i$. Furthermore

$$
\mu_{i}(\mathfrak{p})=\operatorname{Grank} B / \phi(\mathfrak{p})<\infty,
$$

where $\phi$ is the lattice isomorphism from Definition 4.4. In particular, for any such $\mathfrak{p}$ the indecomposable injective $J(\mathfrak{p})$ appears only in the ith term $I^{i}$, where $i:=-\operatorname{Cdim}_{R} A / \mathfrak{p}$.

Proof. This follows from Theorem 5.1 and the one-to-one correspondence between primes ideals and indecomposable injectives [GW, 8.14].

Observe that Corollary 5.3 is similar to Theorem 0.4 with a slightly different hypothesis.

Remark 5.4. In Theorem 0.4 and Corollary 5.3 the $i$ th term in the minimal injective resolution of the Auslander dualizing complex $R$ (as complex of left modules, and, by symmetry, also as complex of right modules) is pure of $\operatorname{Cdim}_{R}=-i$. When this happens we say that $R$ has pure minimal injective resolutions. One of the main results in [YZ2] is that in this case the Cousin complex $\mathrm{E} R$ is a residual complex, and there is an isomorphism $\mathrm{E} R \cong R$ in the derived category $\mathrm{D}\left(\operatorname{Mod} A \otimes B^{\mathrm{op}}\right)$. 
When considered as a complex of left (or right) modules, $\mathrm{E} R$ is a minimal injective complex. Thus there is a decomposition $(\mathrm{E} R)^{i} \cong \bigoplus J(\mathfrak{p})^{\mu_{i}(\mathfrak{p})}$ as left modules, and a decomposition $(\mathrm{E} R)^{i} \cong \bigoplus J(\mathfrak{q})^{\mu_{i}(\mathfrak{q})}$ of right modules, where the sums are over the prime ideals $\mathfrak{p} \subset A$ and $\mathfrak{q} \subset B$ such that $\operatorname{Cdim}_{R ; A} A / \mathfrak{p}=\operatorname{Cdim}_{R ; B^{\text {op }}} B / \mathfrak{q}=-i$.

Proof of Corollary 0.5. Let $d$ be the Krull dimension of A. By [SZ, 3.10] the ring $A$ is Auslander Gorenstein, Kdim-Macaulay of injective dimension $d$. Since $A$ is PI, the dualizing complex $R:=A[d]$ is bifinite (see Lemma 2.1). Then Theorem 0.4 gives the form of $i$ th term of the minimal injective resolution of $R$ as complex of left modules. Note that $\operatorname{Cdim}_{R} M=\operatorname{Kdim} M$ for any finite $A$-module $M$. It remains to show that multiplicities are correct.

The complex $R$ is a pre-balanced dualizing complex over $A$. By [CWZ, 3.9], $A$ admits a balanced dualizing complex, say $R^{\prime}$. By Proposition 4.5(3), $R^{\prime}$ has the trace property for ideals; so Theorem 5.1 holds for $R^{\prime}$. Now by Proposition 2.3 we have $R^{\prime} \cong L \otimes_{A} R$ in $\mathrm{D}\left(\operatorname{Mod} A^{\mathrm{e}}\right)$ for some invertible bimodule $L$. Since $A$ is local the bimodule $L$ is isomorphic to $A^{\sigma}$ for some automorphism $\sigma$. Therefore $R^{\prime} \cong R=A[d]$ in $\mathrm{D}(\operatorname{Mod} A)$, and we deduce that the resolution of $A$ has the correct multiplicities.

Finally we briefly mention that the multiplicity of an injective not of the form $J(\mathfrak{p})$ is usually zero or infinite. In the next proposition we show this for injective hulls of simple modules.

Proposition 5.5. Let $R$ be a dualizing complex over $(A, B)$. Suppose that for every ideal $\mathfrak{b} \varsubsetneqq B$ the quotient ring $B / \mathfrak{b}$ is not right artinian. Let $S$ be a simple $A$-module. If the injective hull $E(S)$ appears in the ith term of the minimal injective resolution of $R$ as complex of A-modules, then its multiplicity is infinite.

Proof. Let $R \rightarrow I$ be the minimal injective resolution of $R$ as complex of $A$ modules. Since $S$ is simple the complex $\operatorname{Hom}_{A}(S, I)$ has zero differentials, and hence $\operatorname{Ext}_{A}^{i}(S, R) \cong \operatorname{Hom}_{A}\left(S, I^{i}\right)$. Define $M:=\operatorname{Ext}_{A}^{i}(S, R)$. If the multiplicity of $E(S)$ in $I^{i}$ is a positive integer, say $n$, then

$$
\operatorname{Hom}_{A}\left(S, I^{i}\right) \cong \operatorname{Hom}_{A}\left(S, S^{n}\right) \cong D^{n}
$$

where $D:=\operatorname{Hom}_{A}(S, S)$ is a division ring. Therefore $M$ is a $\left(D^{\mathrm{op}} \otimes B^{\mathrm{op}}\right)$-module which is finite over $B^{\text {op }}$ and finite length over $D^{\text {op }}$. By Lenagan's lemma [GW, 7.10] $M$ is a finite length $B^{\mathrm{op}}$-module. Let $\mathfrak{b}:=\operatorname{Ann}_{B^{\circ \mathrm{op}}}(M)$. Then $B / \mathfrak{b}$ is right artinian. This contradicts our hypothesis. Therefore the multiplicity of $E(S)$ in $I^{i}$ has to be either 0 or infinite.

\section{EXAmples}

In the first example we show that not every dualizing complex is weakly bifinite.

Example 6.1. Let $C \subset D$ be division rings such that $\operatorname{dim}_{C}$ op $D$ is finite and $\alpha:=\operatorname{dim}_{C} D$ is infinite $[\mathrm{Co}, 5.6 .1]$. Consider the upper triangular matrix ring $A:=\left[\begin{array}{cc}D & D \\ 0 & C\end{array}\right]$. By $[\mathrm{AF}$, Exc. 9 on p. 286] the ring $A$ is two-sided artinian (hence FBN), and by [MR, 7.5.1] the global dimension of $A$ is 1 . So $R:=A$ is a dualizing complex over $A$.

We claim that the dualizing complex $R$ is not Auslander. Let's identify $C$ and $D$ with the two simple quotient rings of $A$. Let $E(D)$ be the injective hull of 
the simple left $A$-module $D$. By [AF, Exercises 8 and 9 on p. 286] the $A$-module $E(D)$ is not finite; indeed, $E(D) / D \cong D \cong C^{(\alpha)}$, a direct sum of $\alpha$ copies of $C$. Pick an $A$-submodule $M \subset E(D)$ of length $l>3$ (the length of $A$ is 3 ). Then any $\phi \in \operatorname{Hom}_{A}(M, A)$ can't be an injection. It follows that $D \subset \operatorname{Ker}(\phi)$. But then the induced homomorphism $\bar{\phi}: M / D \cong C^{l-1} \rightarrow A$ must be zero. We conclude that $\operatorname{Hom}_{A}(M, A)=0$ and hence $\operatorname{Cdim}_{R ; A} M=-1$. On the other hand $\operatorname{Hom}_{A}(D, A) \neq 0$, implying that $\operatorname{Cdim}_{R ; A} D=0$. Since $D \subset M$ this shows $R$ is not Auslander.

The minimal injective resolution of $R$ as left $A$-module is:

$$
0 \rightarrow A \rightarrow E(D)^{2} \rightarrow C^{(\alpha)} \rightarrow 0 .
$$

From this we see that $\operatorname{Hom}_{A}(C, R)=0$ and

$$
\operatorname{Ext}_{A}^{1}(C, R) \cong \operatorname{Hom}_{A}\left(C, C^{(\alpha)}\right) \cong C^{(\alpha)}
$$

as $A^{\mathrm{op}}$-modules. Hence $R$ is not weakly bifinite. Furthermore $R$ does not have trace property for (prime) ideals.

Now suppose $A$ is a finite $\mathbb{k}$-algebra. Then $A^{*}:=\operatorname{Hom}_{\mathbb{k}}(A, \mathbb{k})$ is the rigid dualizing complex over $A$. It is clear that $A^{*}$ is Auslander, bifinite, Cdim-symmetric, and has the trace property for ideals. But usually $A$ has many non-isomorphic dualizing complexes. The next examples, taken from [ASZ, 5.4], show that there exist algebras $A$ and dualizing complexes $R$ over $A$ such that:

(1) $R$ is not Auslander.

(2) $R$ is Auslander, but it does not have the trace property for prime ideals, nor is it Cdim-weakly symmetric.

Example 6.2. Let $A:=\left[\begin{array}{cc}\mathbb{k} & \mathbb{k} \\ 0 & \mathbb{k}\end{array}\right]$. Then $A$ is Auslander regular of global dimension 1 , so $R:=A$ is an Auslander dualizing complex over $A$. Clearly $R$ is bifinite (see Lemma 2.1(1)). Let $\mathbb{k}_{1}$ and $\mathbb{k}_{2}$ be the simple quotient rings of $A$, corresponding to the prime ideals $\mathfrak{p}_{1}:=\left[\begin{array}{cc}0 & \mathbb{k} \\ 0 & \mathbb{k}\end{array}\right]$ and $\mathfrak{p}_{2}:=\left[\begin{array}{cc}\mathbb{k} & \mathbb{k} \\ 0 & 0\end{array}\right]$ respectively. An easy computation shows that $\operatorname{Ext}_{A}^{0}\left(\mathbb{k}_{1}, R\right) \neq 0$ and $\operatorname{Ext}_{A^{\text {op }}}^{0}\left(\mathbb{k}_{1}, R\right)=0$. Hence $R$ is not Cdim-weakly symmetric and not pre-balanced.

Note that $\mathfrak{p}_{1}$, considered as left module, is an injective hull of the simple $A$ module $\mathbb{k}_{1}$. The minimal injective resolution of $A$ as left $A$-module is

$$
0 \rightarrow A \rightarrow \mathfrak{p}_{1} \oplus \mathfrak{p}_{1} \rightarrow \mathbb{k}_{2} \rightarrow 0 .
$$

Hence

$$
\operatorname{RHom}_{A}\left(\mathbb{k}_{1}, R\right) \cong \operatorname{RHom}_{A}\left(\mathbb{k}_{1}, \mathbb{k}_{1}\right)^{2} \cong \mathbb{k}_{1} \oplus \mathbb{k}_{1}
$$

in $\mathrm{D}\left(\operatorname{Mod} \mathbb{k}_{1} \otimes A^{\text {op }}\right)$. Since every dualizing complex over $\mathbb{k}$ is isomorphic to $\mathbb{k}[n]$ for some integer $n$, it follows that $\mathrm{RHom}_{A}\left(\mathbb{k}_{1}, R\right)$ can not be a dualizing complex over $\left(\mathbb{k}_{1}, \mathbb{k}_{1}\right)$ nor over $\left(\mathbb{k}_{1}, \mathbb{k}_{2}\right)$. Therefore by Lemma $4.2 R$ does not have trace property for prime ideals.

Example 6.3. Let $A:=\left[\begin{array}{cc}\mathbb{k} & \mathbb{k}^{n} \\ 0 & \mathbb{k}\end{array}\right]$ for some $n \geq 2$. Then $A$ has global dimension 1 , and $R:=A$ is a dualizing complex over $A$; but $R$ is not Auslander. Like in the previous example, $R$ is bifinite, and does not have the trace property for prime ideals. 
In $[\mathrm{ASZ}, 5.4]$ the following was proved. Consider the ring $A$ of Example 6.3. Let $E$ be the injective hull of $A$ as $A^{\mathrm{op}}$-module. Then $E$ has a "natural" $A$-module structure, but $E$ is not an injective $A$-module. We now prove a general statement, which applies to Example 6.1 as well.

Proposition 6.4. Let $A$ be a noetherian ring of injective dimension 1, which is not Auslander. Let $R:=A$, a dualizing complex over $A$. Assume there is an exact dimension function $\operatorname{dim}$ on $\operatorname{Mod} A$ and $\operatorname{Mod} A^{\text {op }}$ such that $R$ has pure minimal injective resolutions on both sides relative to dim. Then there does not exist an $A$-bimodule $E$ that contains a sub-bimodule isomorphic to $A$, such that $E$ is an injective hull of $A$ on both sides.

Proof. Suppose on the contrary that such a bimodule $E$ exists. Then the bimodule complex

$$
R^{\prime}:=(0 \rightarrow E \rightarrow E / A \rightarrow 0),
$$

with $E$ in degree 0 , is a minimal injective resolution of $R$ on both sides. By assumption $E$ and $E / A$ are dim-pure on both sides. So $R^{\prime}$ is a residual complex over $A$. By $[\mathrm{Ye} 2,2.6]$ the complex $R$ is Auslander $\left(\right.$ and $\left.\operatorname{Cdim}_{R}=\operatorname{dim}\right)$. Hence $A$ is an Auslander ring, a contradiction.

In the next example we discuss some properties of dualizing complexes over Weyl algebras.

Example 6.5. Let $A$ be the $n$th Weyl algebra over $\mathbb{k}$, for some $n \geq 1$. Then $A$ is Auslander regular (and Cohen-Macaulay). Hence the bimodule $A$ is an Auslander (Cohen-Macaulay) dualizing complex over $A$. The rigid dualizing complex over $A$ is $A[2 n]$; this is proved in $[Y e 4,2.6]$ for char $\mathbb{k}=0$, and for positive characteristic it follows from the fact that $A$ is Azumaya over its center and using [YZ2, Theorem 6.2]. By [YZ3], if $R$ is a dualizing complex over $(B, A)$ for some left noetherian ring $B$, then $B$ is Morita equivalent to $A$ and $R \cong P[m]$ for some $m$ and some invertible bimodule $P$. We see that every dualizing complex over $(B, A)$ is Auslander (and Cohen-Macaulay).

Now take $n=1$. When char $\mathbb{k}=0$ the dualizing complex $A[1]$ is pre-balanced, and when char $\mathbb{k}>0$ the complex $A[2]$ is pre-balanced. Thus every dualizing complex over $(B, A)$ is pre-balanced after a suitable shift.

Suppose now char $\mathbb{k}=0$ and $n \geq 2$. By a result of Stafford [St] there is a simple $A$-module of GKdim $>n$ (these are called non-holonomic simples). Using this fact we can show that the dualizing complex $A[n]$ is not pre-balanced. This implies that $P[n]$ is not pre-balanced for any invertible bimodule $P$. For $m \neq n$ the dualizing complex $P[m]$ fails the pre-balanced test for holonomic simple modules. Therefore there is no pre-balanced dualizing complex over $(B, A)$ for any noetherian $\operatorname{ring} B$.

Finally, assume char $\mathbb{k}=0$ and $n \geq 1$. Let $S$ be a simple $A$-module. Then by Proposition 5.5 the multiplicity of $E(S)$ in the $i$ th term of the minimal injective resolution of $A$ as left module is either 0 or infinite.

\section{Miscellaneous}

The results in this section are not directly related to multiplicities of injectives, but they are related to the methods and ideas presented in the previous sections.

Recall that when we say $R$ is a dualizing complex over $(A, B)$ we assume that $A$ is left noetherian and $B$ is right noetherian. The first proposition is a consequence of Lemma 2.5. 
Proposition 7.1. Let $R$ be a dualizing complex over a pair of rings $(A, B)$. Then $A$ is $P I$ if and only if $B$ is PI.

Proof. Assume $B$ is PI; we want to show $A$ is PI too. Since $A$ is left noetherian, it suffices to show that $A / \mathfrak{p}$ is PI for all primes $\mathfrak{p} \subset A$. So choose one such $\mathfrak{p}$. By [YZ1, 1.11] the $i$ th term in the minimal injective resolution of $R$ as complex of $A$-modules contains a nonzero left ideal of $A / \mathfrak{p}$. By Lemma 2.5(3) we know that $L:=\operatorname{Ext}_{A}^{i}(A / \mathfrak{p}, R)$ is not torsion as $(A / \mathfrak{p})$-module. Let $N$ be the torsion submodule of $L$, so that $L / N$ is a nonzero $(A / \mathfrak{p})$ - $B$-bimodule which is torsion-free as $(A / \mathfrak{p})$ module. Pick a nonzero sub-bimodule $M \subset L / N$ which is a faithful $(B / \mathfrak{q})^{\mathrm{op}}$-module for some prime ideal $\mathfrak{q} \subset B$. Let $Q$ be the fraction $\operatorname{ring}$ of $B / \mathfrak{q}$. Since $B$ is PI the simple artinian ring $Q$ is finite over its center $\mathrm{Z}(Q)$. Now $M^{\prime}:=M \otimes_{B / \mathfrak{q}} Q$ is a finite $Q^{\mathrm{op}}$-module, which is also a torsion-free $(A / \mathfrak{p})$-module. We obtain a ring injection $A / \mathfrak{p} \hookrightarrow \operatorname{End}_{Q}\left(M^{\prime}\right)$. Since the latter is a finite $\mathrm{Z}(Q)$-algebra it follows that $A / \mathfrak{p}$ is PI.

The second proposition is an application of the trace property.

Proposition 7.2. Let $R$ be an Auslander dualizing complex over a pair of rings $(A, B)$. Assume $R$ has the trace property for prime ideals. Then $A$ is left FBN if and only if $B$ is right $F B N$.

Proof. Suppose on the contrary that $A$ is left FBN but $B$ is not right FBN. Then there is a prime ideal $\mathfrak{q} \subset B$ and a faithful finite $(B / \mathfrak{q})^{\mathrm{op}}$-module $M$ which is torsion [GW, 8.2]. By the trace property for prime ideals we can assume that $A$ and $B$ are prime rings and $\mathfrak{q}=0$. Since $M$ is a torsion $B^{\text {op }}$-module we have

$$
\operatorname{Cdim}_{R ; B^{\text {op }}} M<\operatorname{Cdim}_{R ; B^{\text {op }}} B .
$$

By the Auslander property we also know that

$$
\operatorname{Cdim}_{R ; A} \operatorname{Ext}_{B^{\text {op }}}^{i}(M, R)<\operatorname{Cdim}_{R ; B^{\text {op }}} B=\operatorname{Cdim}_{R ; A} A
$$

for any $i$. Since $A$ is left FBN, there is a nonzero two-sided ideal $\mathfrak{a} \subset A$ such that $\mathfrak{a} \cdot \operatorname{Ext}_{B^{\text {op }}}^{i}(M, R)=0$ for all $i$.

Now let $N$ be a finite $A$-module which is not faithful. We claim that $\operatorname{Ext}_{A}^{i}(N, R)$ is a non-faithful $B^{\text {op }}$-module for all $i$. By noetherian induction and exact sequences we can reduce to the case when $\mathfrak{p} N=0$ for some nonzero prime ideal $\mathfrak{p} \subset A$. By the trace property there is a nonzero prime ideal $\mathfrak{q} \subset B$ and an induced dualizing complex $\bar{R}$ over $(A / \mathfrak{p}, B / \mathfrak{q})$. Then

$$
\operatorname{Ext}_{A}^{i}(N, R) \cong \operatorname{Ext}_{A / \mathfrak{p}}^{i}(N, \bar{R})
$$

as $B^{\mathrm{op}}$-modules. It follows that $\operatorname{Ext}_{A}^{i}(N, R) \cdot \mathfrak{q}=0$.

Applying the above claim to $N:=\operatorname{Ext}_{B^{\text {op }}}^{j}(M, R)$ one sees that the $B^{\mathrm{op}}$-module $\operatorname{Ext}_{A}^{i}\left(\operatorname{Ext}_{B^{\text {op }}}^{j}(M, R), R\right)$ is not faithful for all $i$ and $j$. Finally the double-Ext spectral sequence $[\mathrm{YZ1}, 1.7]$ shows that $M$ is not faithful, a contradiction.

If $A=B$ we have the following corollary.

Corollary 7.3. Let $A$ be a noetherian algebra. Suppose $A$ admits an Auslander dualizing complex with the trace property for prime ideals. Then $A$ is left FBN if and only if it is right FBN. 
It is unknown whether every noetherian left FBN ring is also right FBN [GW, p. 132].

Finally we show that the "Cdim-symmetric" property is sometimes a consequence of other properties. The following proposition is an analogue of [SZ, 3.10]. The proof of this is also very similar to the proof of [SZ, 3.10]. For the convenience of reader we include it here.

Proposition 7.4. Let $A$ and $B$ be FBN rings and let $R$ be a pre-balanced, weakly bifinite dualizing complex over $(A, B)$. Then:

(1) $R$ is Auslander.

(2) $\operatorname{Cdim}_{R ; A} M=\operatorname{Kdim} M$ for all finite $A$-modules $M$, and likewise for all finite $B^{\mathrm{op}}$-modules. As a consequence, $R$ is Cdim-symmetric.

Proof. We will prove the following four statements by induction on the Krull dimension of the finite $A$-module $M$.

(a) $\operatorname{Ext}_{A}^{i}(M, R)=0$ if $i>0$ or $i<-\operatorname{Kdim} M$.

(b) $\operatorname{Cdim}_{R ; A} M=\operatorname{Kdim} M$.

(c) $\operatorname{Kdim}_{\operatorname{Ext}}^{j}(M, R)=\operatorname{Kdim} M$ where $j:=j_{R}(M)$.

(d) For every $i \geq j_{R}(M)$ one has $\operatorname{Kdim}_{\operatorname{Ext}}^{i}(M, R) \leq-i$.

By symmetry these statements will also hold after replacing $A$ with $B^{\text {op }}$. The Auslander condition will then follow.

If $\operatorname{Kdim} M=0$ then $M$ has finite length, and the problem can be reduced to the case when $M$ is simple, in which case the assertions follow from the pre-balanced hypothesis.

Now suppose $\operatorname{Kdim} M=d>0$, and assume the assertions hold for all modules of lower Krull dimension. Using noetherian induction and [SZ, Lemma 2.1], it suffices to check the case $M=A / \mathfrak{p}$ for some prime ideal $\mathfrak{p}$. Let $\bar{A}$ be the quotient $\operatorname{ring} A / \mathfrak{p}$. For every regular element $u \in \bar{A}$ right multiplication gives a short exact sequence

$$
0 \rightarrow \bar{A} \rightarrow \bar{A} \rightarrow \bar{A} / \bar{A} u \rightarrow 0,
$$

which induces a long exact sequence

$$
\operatorname{Ext}_{A}^{i}(\bar{A} / \bar{A} u, R) \rightarrow \operatorname{Ext}_{A}^{i}(\bar{A}, R) \rightarrow \operatorname{Ext}_{A}^{i}(\bar{A}, R) \rightarrow \operatorname{Ext}_{A}^{i+1}(\bar{A} / \bar{A} u, R) .
$$

Observe that $\operatorname{Kdim} \bar{A} / \bar{A} u<d$. Write $E=\operatorname{Ext}_{A}^{i}(\bar{A}, R)$ and let $Q$ be the fraction ring of $\bar{A}$.

Suppose $i>0$ or $i<-d$. In this case the two end terms in (7.5) are zero by the induction hypothesis, so left multiplication by $u$ on $E$ is an isomorphism. Since $Q$ is obtained by inverting all such regular elements $u$ it follows that $E$ is a $Q$-module. By the "weakly bifinite" hypothesis $E$ is a finite $\bar{A}$-module. If $E \neq 0$ then $Q$ is a submodule of a finite direct sum of copies of $E$. This implies that $Q$ is a finite $\bar{A}$-module, and hence $Q=\bar{A} v^{-1}$ for some regular element $v \in \bar{A}$. Then $Q=Q v=\bar{A} v^{-1} v=\bar{A}$, so $\mathfrak{p}$ is a maximal ideal $\operatorname{Kdim} \bar{A}=0$, which is a contradiction. Therefore $E=0$; this is statement (a).

Now suppose $-d \leq i \leq 0$. By the inductive hypothesis

$$
\mathrm{Kdim}_{\operatorname{Ext}}^{i+1}(\bar{A} / \bar{A} u, R) \leq-i-1 \text {. }
$$

The long exact sequence 7.5 shows that $E / u E$ is a submodule of $\operatorname{Ext}_{A}^{i+1}(\bar{A} / \bar{A} u, R)$, so

$$
\operatorname{Cdim}_{R ; B^{\mathrm{op}}} E / u E \leq-i-1
$$


From the induction hypothesis (this time $B^{\mathrm{op}}$-modules) we see that $\mathrm{K} \operatorname{dim} E / u E \leq$ $-i-1$. Since this holds for all regular elements $u$, the argument in [SZ, p. 1003] shows that $\operatorname{Kdim} E \leq-i$. This is statement (d).

Finally we look at the case $i=-d$. The last paragraph shows that $\operatorname{Kdim} E \leq d$. We want to show there is equality. If not then $\operatorname{Kdim} E<d$, and by the previous

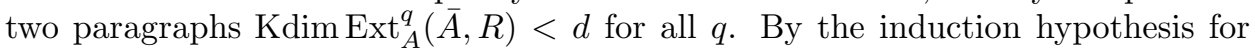
$B^{\mathrm{op}}$-modules we see that $\operatorname{Ext}_{B^{\mathrm{op}}}^{p}\left(\operatorname{Ext}_{A}^{q}(\bar{A}, R), R\right)$ has Krull dimension less than $d$ for all $p, q$. The double-Ext spectral sequence [YZ2, 1.7] implies that $\operatorname{Kdim} \bar{A}<d$, and this is a contradiction. Therefore $\operatorname{Kdim}_{\operatorname{Ext}}^{-}{ }_{A}^{-d}(\bar{A}, R)=d$, and as a consequence we obtain statements (b) and (c).

\section{REFERENCES}

[AF] F.W. Anderson and K.R. Fuller, "Rings and Categories of Modules," Second edition, Graduate Texts in Mathematics 13, Springer-Verlag, New York, 1992.

[ASZ] K. Ajitabh, S.P. Smith and J.J. Zhang, Auslander-Gorenstein rings, Comm. Algebra 26 (1998), no. 7, 2159-2180.

[BM] G. Barou and M.-P. Malliavin, Sur la résolution injective minimale de l'algèbre enveloppante d'une algébre de Lie résoluble. (French) J. Pure Appl. Algebra 37 (1985), no. 1, $1-25$.

[Br] K.A. Brown, Fully bounded rings of finite injective dimension, Quart. J. Math. Oxford Ser., 41 (1990), 1-13.

[BH] K.A. Brown and C.R. Hajarnavis, Injectively homogeneous rings, J. Pure Appl. Algebra 51 (1988), no. 1-2, 65-77.

[BL] K.A. Brown and T. Levasseur, Cohomology of bimodules over enveloping algebras, Math. Z. 189 (1985), no. 3, 393-413.

[CWZ] D. Chan, Q.-S. Wu and J. J. Zhang, Pre-balanced dualizing complexes, Israel J. Math. 132 (2002), 285-314.

[Co] P.M. Cohn, "Skew field constructions" London Math. Soc. Lecture Note Series, No. 27, Cambridge University Press, 1977.

[GL] K.R. Goodearl and T.H. Lenagan, Catenarity in quantum algebras, J. Pure Appl. Algebra 111 (1996), no. 1-3, 123-142.

[GW] K.R. Goodearl and R.B. Warfield, Jr., "An introduction to noncommutative Noetherian rings", London Mathematical Society Student Texts, 16. Cambridge University Press, Cambridge-New York, 1989.

[Mal] M.-P. Malliavin, Modules sans torsion et modules injectifs sur les algèbres de Lie résolubles. (French) J. Algebra 83 (1983), no. 1, 126-157.

[MR] J.C. McConnell and J.C. Robson, "Noncommutative Noetherian Rings," Wiley, Chichester, 1987.

[Ri1] J. Rickard, Morita theory for derived categories, J. London Math. Soc. (2) 39 (1989), no. $3,436-456$.

[Ri2] J. Rickard, Derived equivalences as derived functors, J. London Math. Soc. (2) 43 (1991), no. $1,37-48$.

[St] J. T. Stafford, Nonholonomic modules over Weyl algebras and enveloping algebras, Invent. Math. 79 (1985), no. 3, 619-638.

[SZ] J.T. Stafford and J.J. Zhang, Homological properties of (graded) noetherian PI rings, J. Algebra 168 (1994), 988-1026.

[VdB] M. Van den Bergh, Existence theorems for dualizing complexes over non-commutative graded and filtered ring, J. Algebra 195 (1997), no. 2, 662-679.

[WZ1] Q. Wu and J.J. Zhang, Dualizing complexes over noncommutative local rings, J. Algebra 239 (2001), 513-548.

[WZ2] Q. Wu and J.J. Zhang, Homological identities for noncommutative rings, J. Algebra 242 (2001), 516-535.

[Ye1] A. Yekutieli, Dualizing complexes over noncommutative graded algebras, J. Algebra 153 (1992), 41-84. 
[Ye2] A. Yekutieli, The residue complex of a noncommutative graded algebra, J. Algebra 186 (1996), 522-543.

[Ye3] A. Yekutieli, Dualizing complexes, Morita equivalence and the derived Picard group of a ring, J. London Math. Soc. (2) 60 (1999), no. 3, 723-746.

[Ye4] A. Yekutieli, The rigid dualizing complex of a universal enveloping algebra, J. Pure Appl. Algebra 150 (2000), no. 1, 85-93.

[YZ1] A. Yekutieli and J.J. Zhang, Rings with Auslander dualizing complexes, J. Algebra 213 (1999), no. 1, 1-51.

[YZ2] A. Yekutieli and J.J. Zhang, Residue Complexes over Noncommutative Rings, J. Algebra 259 (2003), 451-493.

[YZ3] A. Yekutieli and J.J. Zhang, Dualizing complexes and tilting complexes over simple rings, J. Algebra 256 (2002), 556-567.

Department of Mathematics, Ben Gurion University, Be'er Sheva 84105, ISRAeL

E-mail address: amyekut@math.bgu.ac.il

Department of Mathematics, University of Washington, Seattle, WA 98195, USA

E-mail address: zhang@math.washington.edu 